\title{
Time-Dispersion Simulation for Microcellular Systems in Urban Environments
}

\author{
Shiun-Chi Jan and Shyh-Kang Jeng $\ddagger$ \\ Department of Electrical Engineering, National Taiwan University, \\ Taipei 10617, Taiwan
}

\section{Introduction}

Over the past decade the mobile communications industry has experienced phenomenal growth. As the number of communication devices continue to increase, high-capacity microcellular communication networks will grow. Fairly extensive work has been done to characterize narrow band propagation in terms of the mean signal strength and the fading characteristics. It's still necessary to adequately quantify the time-dispersion characteristics for wideband communication system, whose data rate exceeds the coherent bandwidth. But only few papers focused on the time-dispersion characteristics[1,2]. For microcells, propagation modeling must take into account the exact positions, orientations, and electrical properties of individual buildings. Wideband channel have been simulated in the urban environments using the modified SBR/Image method[4], which is a site-specific prediction method. We sought to investigate the effects on the time dispersion characteristics of the channel to complement an earlier study of propagation loss characteristics.

Theory

The impulse response of a multipath channel can be modeled mathematically as $[3]$

$$
h(t)=\sum_{i=0}^{I-1} E_{i} \delta\left(t-\tau_{i}\right)
$$

where $I$ is number of multipath components, and $\delta 0$ is the Dirac delta function. The ith path is characterized by electrical field strength $E_{i}$, and its propagation delay $\tau_{i}$. For a measurement system, there is a finite time-resolution. If signals arrive through different paths within a time interval smaller than the resolution limited by the measurement system, they cannot be distinguished. They are considered as a single path component with a common delay and their signal strengths are added coherently. This is the same as the discrete-time impulse response model used in [3]. The time axis is divided into small time bins according to the resolution and is labeled form bin 0 .

In this paper, we use the modified SBR/Image method along with the UTD theory to obtain the electrical field strength $E_{i}$ and time delay $\tau_{i}$ in each bin. The modified SBR/Image method has been briefly presented in Section 2 of [4]. If the ray is with $L$ reflections, $M$ transmissions and $N$ diffractions, the field at the receiver is given by

$$
\bar{E}^{r}=\bar{E}_{0} \cdot \prod_{l=1}^{L} \overline{\overline{R_{l}}} \cdot \prod_{m=1}^{M} \overline{\overline{T_{m}}} \cdot \prod_{n=1}^{N} A_{n} \overline{\overline{D_{n}}} \cdot e^{-\jmath\left(k_{m}-k\right) d_{m}} \cdot \frac{e^{-\jmath k s}}{s}
$$

0-7803-4178-3/97/\$10.00 ㅇ 1997 IEEE 
where $\bar{E}_{0}$ is the electric field at the reference point; $\overline{\overline{R_{l}}}$ is the dyadic reflection coefficient at the 1'th reflection point; $\overline{\bar{T}_{m}}$ is the dyadic transmission coeffcient at the m'th transmission point; $A_{n}$ is the divergence factor at the $n$ 'th diffraction point; $\overline{\overline{D_{n}}}$ is the dyadic diffraction coefficient at the $n$ 'th diffraction point; $k_{m}$ is the wave number in the m'th transmission medium; $d_{m}$ is the ray path length in the m'th transmission medium; $s$ is the ray path length from the transmitter to the receiver; and $k$ is the wave number in free space. From $(2)$ and ray racing parameters, $E_{i}$ and $\tau_{i}$ for each bin can be determined for studying the time-dispersion characteristics.

Results

Through the modified SBR/Image method and UTD formulation, simulations were conducted to determine the rms delay spread in urban environments. A street scene with tall buildings on both sides of the street was considered. The main street and the side street is $20 \mathrm{~m}$ wide and each block is $75 \mathrm{~m}$ long. The ground were modeled as a dielectric halfspace with $\epsilon_{r}=15$ for the relative permittivity, and $\sigma=0.0075$ for the conductivity. The relative permittivity and the conductivity of building walls are $\epsilon_{r}=2$ and $\sigma=0.0075$, respectively. In the paper by Feuerstein[1], an overbound model of rms delay spread on propagation loss was proposed. Figure 1 shows rms delay spread versus propagation loss from simulation results and from the overbound model by measurement data [1]. It's shown that the simulation results are much lower than the overbound model. In more realistic environments, the wall separation is not always uniform. A street scene, whose wall separation is a random variable with normal distribution[2], were simulated and the result is also shown in Fig. 1. It's found that the rms delay spread is wider and closer to the overbound model. It's observed that far reflections contribute most to the wider rms delay spread. Figure 2 shows the rms delay spread for different bandwidths. Due to the limited resolution, the better the resolution, the narrower the maximum rms spread. Figure 3 shows the overbound model as well as simulations based on different relative permittivity of walls (from $2-8$ ). From the figure, we find strong reflection leads to a higher rms delay spread. Figure 4 shows the effect of different routes of receiver motion. It's shown that rms delay become wider in route $\mathrm{A}$ and narrower in route $\mathrm{D}$.

\section{Conclusion}

Using the modified SBR/Image method, we have investigated the effects of street layout, base station location and the electrical characteristics of buildings on time dispersion characteristics. Precise building layout, accurate transceiver locations, and suitable electrical characteristics are important for the simulation of time dispersion characteristics. More simulation results will be presented in the conference.

Acknowledgment This work was supported by the Telecommunication Laboratory, Directorate General of Telecommunications, under Grant TL-862203; and by National Science Council, under Grant NSC-86-2221-E002-037.

\section{References}

[1 ] M. J. Feuerstein, K. L. Blackard, T. S. Rappaport, S. Y. Seidel, and H. H. $\mathrm{Xia}$, "Path loss, delay spread, and outage models as functions of antenna height for microcellular system design," IEEE Trans. Veh. Technol., vol. VT-43, no. 3, pp. 487-498, Aug. 1994 
[2] U. Kauschke, "Propagation and system performance simulations for the short range DECT system in microcellular urban roads," IEEE Trans. Veh. Technol., vol. VT-44, no. 2, pp. 253-260, May 1995.

[3 ] G. L. Turin, "Introduction to spread-spectrum antimultipath techniques and their application to urban digital radio," Proc. IEEE, vol. 68, pp. 328-353, Mar. 1980.

[4 ] S. C. Jan and S. K. Jeng, "A novel propagation modeling for microcellular communications in urban environments," to be published in IEEE Trans. Vehicular Technology.

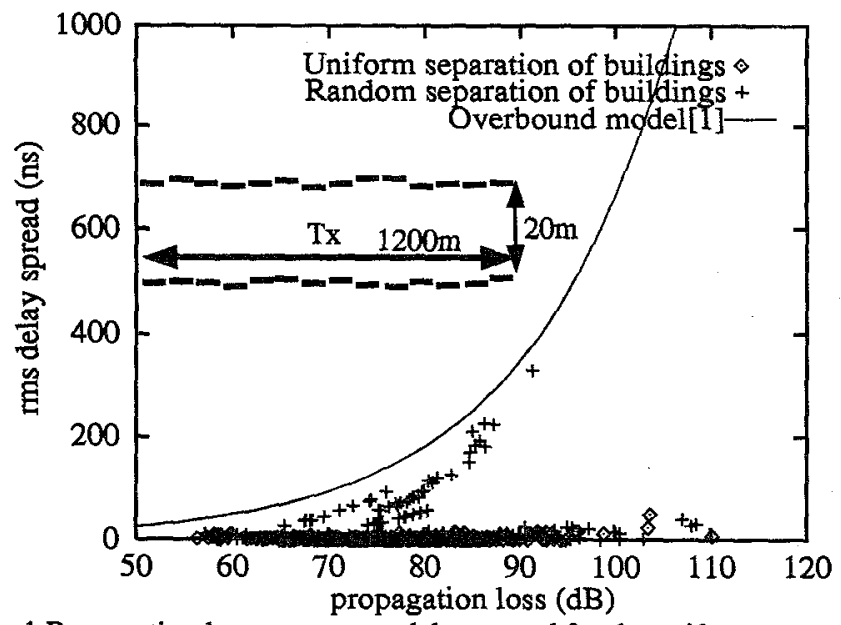

Figure 1 Propagation loss versus rms delay spread for the uniform separation of buildings case and the random separation of buildings case (normal distribution).

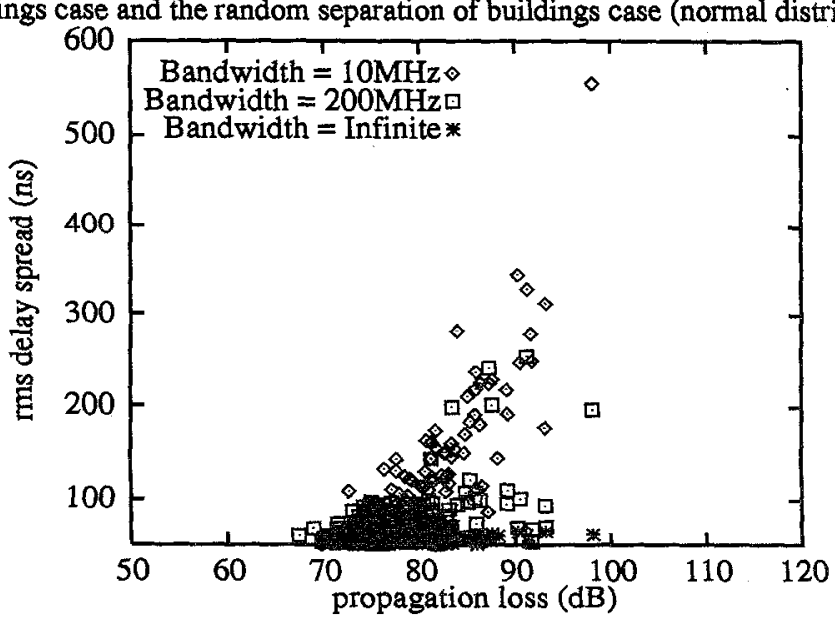

Figure 2 Propagation loss versus rms delay spread for differenttest bandwidths. 


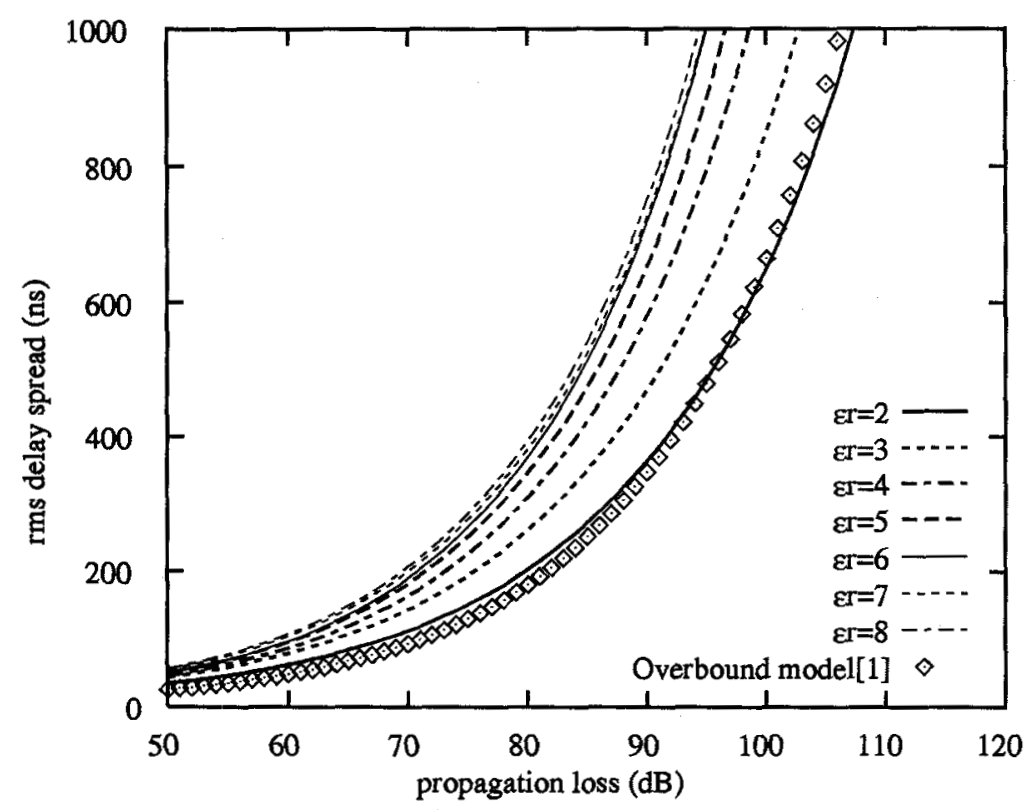

Figure 3 Rms delay spread overbounds on propagation loss for different relative permittivity of building walls.

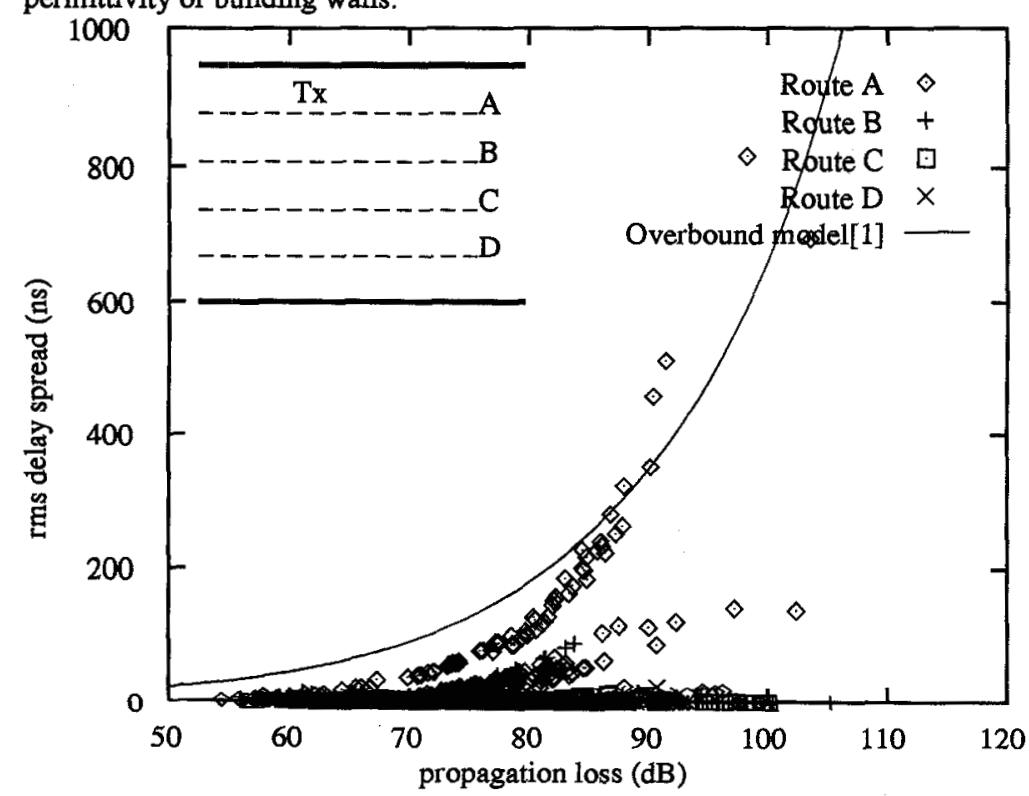

Figure 4 Propagation loss versus rms delay spread for different test routes (transmitting base station is located on one side of the road). 